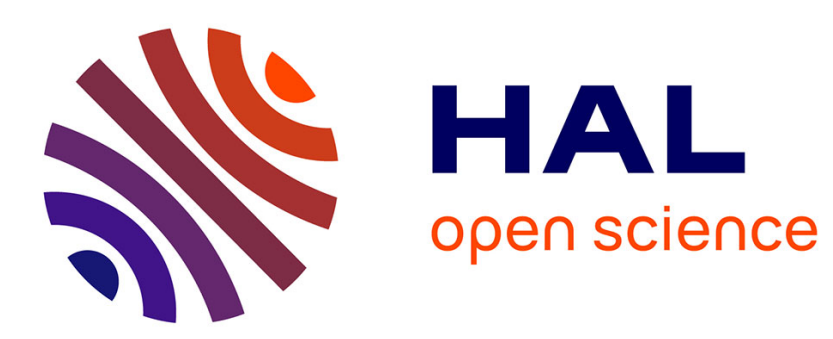

\title{
L'altérité à l'épreuve de l'ubiquité informationnelle
}

David Pucheu

\section{To cite this version:}

David Pucheu. L'altérité à l'épreuve de l'ubiquité informationnelle. Hermès, La Revue - Cognition, communication, politique, 2014, 1 (68), pp.115-122. hal-01805976

\section{HAL Id: hal-01805976 https://hal.science/hal-01805976}

Submitted on 13 Jan 2021

HAL is a multi-disciplinary open access archive for the deposit and dissemination of scientific research documents, whether they are published or not. The documents may come from teaching and research institutions in France or abroad, or from public or private research centers.
L'archive ouverte pluridisciplinaire HAL, est destinée au dépôt et à la diffusion de documents scientifiques de niveau recherche, publiés ou non, émanant des établissements d'enseignement et de recherche français ou étrangers, des laboratoires publics ou privés. 


\section{L'ALTÉRITÉ À L'ÉPREUVE DE L'UBIQUITÉ INFORMATIONNELLE}

\section{David Pucheu}

C.N.R.S. Editions | « Hermès, La Revue »

2014/1 n 68 | pages 115 à 122

ISSN 0767-9513

ISBN 9782271080745

Article disponible en ligne à l'adresse :

https://www.cairn.info/revue-hermes-la-revue-2014-1-page-115.htm

Distribution électronique Cairn.info pour C.N.R.S. Editions.

(C) C.N.R.S. Editions. Tous droits réservés pour tous pays.

La reproduction ou représentation de cet article, notamment par photocopie, n'est autorisée que dans les limites des conditions générales d'utilisation du site ou, le cas échéant, des conditions générales de la licence souscrite par votre établissement. Toute autre reproduction ou représentation, en tout ou partie, sous quelque forme et de quelque manière que ce soit, est interdite sauf accord préalable et écrit de l'éditeur, en dehors des cas prévus par la législation en vigueur en France. Il est précisé que son stockage dans une base de données est également interdit. 


\section{David Pucheu}

EA Mica - université Bordeaux-Montaigne

\section{L'altérité à l'épreuve de l'ubiquité informationnelle}

Depuis plus d'une vingtaine d'années maintenant, d'innombrables consultants et technologues - pour la plupart nord-américains - annoncent sur un ton prophétique l'émergence d'un monde médiaté par une infinité de dispositifs numériques disséminés dans notre environnement, nos vêtements, nos corps et tous les objets de notre vie quotidienne; des dispositifs tous interconnectés en réseau, pour la plupart invisibles à la conscience de leurs usagers, laissant entrevoir une infrastructure globale d'information et de communication animée par de puissants algorithmes de calcul; une informatique dite "ubiquitaire» destinée à se "dissoudre dans la trame de notre vie quotidienne au point de ne plus en être discernable» (Weiser, 1991); une ère "post-PC» dans laquelle la puissance du traitement automatique de l'information jusqu'alors contenue dans la boîte incubatrice du personal computer serait appelée à se répandre dans le moindre interstice de nos relations avec le monde.

En s'insinuant au fondement de toute relation avec une extériorité, qu'elle soit humaine, naturelle ou matérielle, l'informatique ubiquitaire et ses innombrables avatars (informatique "pervasive», "omniprésente», "proactive», intelligence "ambiante», etc.) promet de liquider les incertitudes et de rectifier l'incomplétude qui préside à nos rap- ports protéiformes avec le monde. Assurer l'opérationnalité des relations entre l'homme et le monde, tel pourrait être le leitmotiv de cette informatique du futur prophétisée à la fin des années 1980 par le défunt directeur de recherche du Xerox PARC (Palo Alto Research Center) Mark Weiser.

Les questions que soulèvent ces visions prospectivistes d'un véritable "écosystème numérique», dont on observe aujourd'hui des manifestations latentes, sont nombreuses et interrogent indirectement presque tout le faisceau des registres de l'expérience humaine. Cette contribution ne vise pas à balayer l'ensemble des problématiques inhérentes à l'hypothétique émergence d'une informatique ubiquitaire généralisée dont on peut douter qu'elle prendra la forme imaginée par ses promoteurs, mais davantage à tracer la genèse de ce "projet» pour en comprendre les fondements à la fois technologiques et symboliques, qui semblent de concert ordonner une nouvelle économie de l'altérité au monde et à l'autre.

\section{Le projet ubiquitaire}

Ce que je souhaiterais désigner ici par «projet ubiquitaire» consiste en un vaste ensemble de programmes 
de recherche et développement dans le domaine des technologies de traitement de l'information. Un ensemble de programmes qui, malgré leur apparente hétérogénéité, me semble concourir à une même entreprise sociotechnique. Outre des considérations purement technologiques, ce projet s'appuie sur une abondante littérature de vulgarisation, une véritable mise en récit de la technique qui inscrit son dessein comme horizon d'une «société de l'information» en route vers son ultime accomplissement (Dourish et Bell, 2011). C’est précisément cette dynamique et cette tension entre développement technologique et mise en récit de la technique qui me semble attester d'un objectif et d'une intentionnalité commune justifiant l'usage du terme "projet». C'est en tout cas ce que met en lumière l'analyse des discours accompagnant ces évolutions technologiques, depuis les premiers écrits de Mark Weiser jusqu'à leurs plus récents développements (Rheingold, 2002; Greenfield, 2007; O'Reilly et Battelle, 2009) à partir desquels on peut clairement dégager un système cohérent de représentations symboliques partagées (Pucheu, 2013).

\section{Effacer l'interface}

Inauguré au début des années 1990 par les travaux pionniers du PARC, le projet ubiquitaire s'articule autour d'une redéfinition en profondeur des modalités de l'interaction homme-machine ${ }^{1}$ (IHM) entraînant avec elle un véritable bouleversement paradigmatique au sein des computer sciences. C'est dans les écrits de Weiser qu'on en trouve la formulation la plus explicite, et notamment dans son article séminal de 1991, "The Computer For the 21st Century». Fustigeant le technocentrisme qui présidait au développement de l'ordinateur personnel (dont le PARC fut pourtant l'un des acteurs incontournable!), Weiser propose de substituer aux traditionnelles interfaces de commande informatique (le clavier, la souris associés aux interfaces graphiques) de multiples interfaces «invisibles» disséminées dans notre environnement. "L'informatique ubiquitaire - écrivait Weiser - envisage un monde physique enrichi et invisiblement entrelacé de capteurs, d'actuateurs, d'écrans et d'éléments computationnels embarqués "sans couture" (seamlessly) dans les objets de nos vies quotidiennes et connectés dans un réseau continu et persistant.» (Weiser, Gold et Brown, 1999)

Cette «invisibilité» qui préside au projet ubiquitaire ne désigne pas tant une invisibilité matérielle que fonctionnelle, où les opérations de traitement automatisé de l'information effectuées par une multitude d'ordinateurs se retireraient "en périphérie de nos consciences» (Weiser, 1991) pour s'inscrire pleinement dans le déroulement itératif de nos (inter)actions avec et sur le monde environnant. Il s'agit en d'autres termes de faire disparaître l'interface, d'effacer l'outil en l'intégrant au plus près de nos conditions d'être au monde pour flirter avec un jargon plus phénoménologique. «Les technologies les plus puissantes sont celles qui disparaissent» écrivait Weiser (1991): c'est cette idée qui constitue la pierre angulaire du projet ubiquitaire et qui va trouver une incroyable fortune dans le champ de l'IHM. Informatique « diffuse», " pervasive», intelligence « ambiante» n'en sont que des variations sémantiques visant à caractériser cette nature "éthérée ${ }^{2}$ » du processus de traitement de l'information. C'est aussi d'une certaine manière cette invisibilité fonctionnelle qui alimente aujourd'hui l'inflation du préfixe «smart-» pour qualifier la nature dynamique et communicante des objets (les smart objects), des choses (smart city, smart community, smart politics) et des dispositifs technologiques (smartphone, smartTV, smartgrid) qui participent à donner corps au projet ubiquitaire.

\section{Vers une technologie anthropocentrée}

C'est sur un ton rédempteur que se déploie la rhétorique du projet ubiquitaire ( $c f$. Cooper et Bowers, 1995) : le 
clavier, la souris et l'écran qui partagent si peu avec notre corporéité, associés à la complexité inhérente de l'informatique (Norman, 1998), ont dramatiquement étouffé les potentialités des technologies de l'information (Weiser, 1991) condamnant l'usager à n'être qu'un "opérateur» de la machine.

L'informatique ubiquitaire, au contraire, s'affiche comme une technologie human centered asservie à notre volonté, actualisant nos intentions et nos désirs. Elle ne demanderait à son utilisateur aucun investissement cognitif et agirait même, dans une logique prédictive, à un stade précognitif: des machines qui s'adapteraient «à l'environnement humain au lieu de forcer les humains à s'adapter à elles, rendant l'usage de l'informatique aussi agréable («refreshing») que faire une ballade dans les bois» (Ibid.).

Cette "adaptabilité» des dispositifs informatiques $\mathrm{au}$ "monde humain» renvoie à la vision d'un environnement «augmenté» de capacités computationnelles réactif au moindre de nos comportements: une informatique «anthropocentrée» dont le mécanisme tout entier s'articulerait autour de l'intentionnalité anticipée de son usager. La traduction de cette téléologie inscrite, selon Weiser (1994), au plus profond de l'évolution technologique de l'informatique est l'émergence d'une informatique toujours plus «sensible» au contexte de son usager (contextaware computing) - la notion de contexte devant être ici envisagée sous de multiples angles selon qu'elle soit centrée sur la localisation physique de l'utilisateur; sur sa situation (ses relations de proximité spatiale et préférentielle) avec les objets et les autres utilisateurs qui l'entourent; sur son identification; ou encore sur ses comportements physiologiques in situ. Autant de données contextuelles extraites par des capteurs qui permettraient à des actuateurs, partie intégrante des dispositifs ubiquitaires, d'opérer des prises de décision automatisées sur la base d'un traitement algorithmique décentralisé.

Mais s'entrecroisent ici plusieurs niveaux d'analyse: à la situation isolée d'un utilisateur s'agrège la collecte massive et systématique des "traces» (aussi triviales ou sensibles soient-elles) laissées par des «quidams» dans le cours de leur vie quotidienne, permettant ainsi d'élaborer des patterns identitaires, comportementaux et contextuels toujours plus fins. Le traitement algorithmique (le data mining) du «big data» - qui désigne cette masse de données exponentielle stockée dans de gigantesques fermes de serveurs - constituerait progressivement "l'intelligence» distribuée de ces systèmes capables d'inférer, à partir d'une situation donnée, des «schémas prédictifs» de nos comportements futurs.

La trajectoire empruntée ces dernières années par l'industrie informatique semble clairement suivre les grandes orientations de ce projet. Que l'on songe à la démultiplication des appareils mobiles; à l'externalisation massive des données (le cloud computing) dans de gigantesque "datawarehouse»; à la généralisation des réseaux de télécommunication sans fil ou encore au déploiement exponentiel des puces RFID dans les objets de nos vies quotidiennes (l'internet des objets) ${ }^{3}$ : toutes ces évolutions technologiques dessinent les contours de cet écosystème numérique ubiquitaire. Le développement et la démultiplication des interfaces post-PC (réalité augmentée, contrôle vocal, surfaces interactives, interfaces tactiles, gestuelles, kinesthésiques, etc.) dans notre paysage technologique semblent également concourir à ce dessein. Ces innovations, principalement irradiées à partir des centres de recherche et de développement des technologies de l'information aux États-Unis (le Massachusetts Institute of Technology, l'université Stanford ou le Xerox PARC pour ne citer qu'eux), sont également portées par des discours et des récits. Ces récits, largement diffusés dans la communauté des ingénieurs informatiques, le sont aussi au travers de nombreux bestsellers ou revues de vulgarisation publiés par des consultants, futurologues ou par les directeurs de recherche technologique eux-mêmes. C'est à cette construction narrative que nous voudrions nous intéresser ici. 


\section{Une nouvelle économie de l'altérité}

Le projet ubiquitaire, on le voit bien, ne propose pas simplement de repenser notre rapport à la machine: à travers ce renouvellement de l'interaction homme-machine, c'est un mouvement de fond bien plus vaste qui se fait jour. Nos relations désormais «augmentées» au monde, aux choses et aux «autres» qui le peuplent, se trouveraient inéluctablement affectées. Mon propos n'est pas tant ici de questionner ces conséquences encore incertaines que d'interroger les imaginaires de la communication et de l'altérité à partir desquels s'organisent les croyances innervant ce projet sociotechnique.

\section{Un «holisme réticulaire»}

En traçant les contours d'un environnement intégralement médiaté par des dispositifs technologiques, le projet ubiquitaire alimente le fantasme d'une «interrelationalité totale du monde» (Baudrillard, 1968): les êtres, au même titre que les objets et les choses qui l'habitent, se trouveraient inéluctablement immergés dans ce que nous pourrions appeler un «holisme réticulaire». Cette totalité unifiée par les réseaux invisibles des dispositifs ubiquitaires - colonisant progressivement la matérialité de notre environnement et de nos corps dans un rapport toujours plus intime - fond et confond les registres humains et artificiels dans une sorte de monade informationnelle.

Comme le suggère Agustin Arraya (1995) dans une virulente critique de l'informatique ubiquitaire: «Par ce tissage des extensions de nous-même vers notre environnement: des parties significatives du monde perdent d'importants aspects de leur altérité («otherness») et l'environnement considéré comme une totalité tend à devenir de plus en plus un artefact subalterne ( a subservient arte- fact»). Cet artefact devenant le monde qui nous entoure immédiatement est presque entièrement "nous" plutôt qu'un énigmatique "autre" ».

L'interconnectivité généralisée des hommes, des objets et de leur environnement par le truchement des technologies ubiquitaires semble à nouveau sur le point d'envelopper le monde humain dans ce que Simondon appelait la «réticulation magique primitive» (Simondon, 1989). Comme le souligne Claude Savary (2000), «prévaut pour cette situation une adhérence au monde qui ne comporte pas, pour l'agent humain et son monde humain, de distinction entre l'objectif et le subjectif, et qui ne comporte pas non plus, pour l'expérience de la nature faite par l'agent humain, de distinction entre le figural - une nature fragmentée en objets - et les qualités de fond - les pouvoirs, les puissances».

Cette indifférenciation des êtres et des choses, tous traversés par une même force vitale qui caractérisait ce mode primitif d'être au monde comme dans l'animisme, ne serait plus entretenue par une quelconque ignorance des phénomènes qui animent le monde. Bien au contraire: c'est l'hypothèse d'une absolue connaissance des phénomènes, portée par le dévoilement de leur structure informationnelle élémentaire, qui laisse entrevoir la maîtrise et le contrôle sans limite d'une création désormais expurgée de ce qui lui restait d'animation occulte (Gauchet, 1985). Le déploiement d'une informatique "omniprésente», sorte de "peau électronique» (Gross, 1999) superposée au réel, pose les conditions de possibilité d'une telle maîtrise: «Nous aurons réussi - écrivait le directeur du consortium Things That Think du MIT - lorsque les ordinateurs auront disparu et que le monde sera devenu notre interface.» (Gershenfeld, 1999)

La fusion des mondes physique et numérique garantie par l'intégration des dispositifs ubiquitaires transformerait ainsi progressivement la moindre de nos interactions avec notre environnement matériel et cognitif en «expérience utilisateur ${ }^{4} »$. La déréliction de l'homme «jeté dans 
le monde», dans une création finalement étrangère à son existence primordiale, ne serait plus une fatalité: tout y serait désormais à son image, à l'image de sa conscience. Comme le soulignait déjà Baudrillard (1968) en voyant naître l'idéologie cybernétique: «ce ne sont plus ses gestes, son énergie, ses besoins, l'image de son corps que l'homme projette dans les objets automatisés, c'est l'autonomie de sa conscience, son pouvoir de contrôle, son individualité propre, l'idée de sa personne».

\section{L'énigme de l'Autre enfin dévoilée}

Les hommes, nous l'avons dit, enveloppés dans cette monade informationnelle qui n'est pas sans faire écho aux premiers écrits de la cybernétique, se trouvent assignés à un «rôle intermédiaire» dans l'ordre de la création : celui d'un corps-interface traversé par les flux informationnels constants qui animent notre monde. «Être vivant - écrivait déjà le père de la cybernétique en 1950 -, c'est participer à un courant continu d'influences venant du monde extérieur et d'actes agissant sur celui-ci, dans lequel nous ne représentons qu'un stade intermédiaire.» (Wiener, 1971) Plus que jamais, cette visée ontologique destinée à caractériser une nature humaine réduite à la somme indifférenciée des comportements informationnels de l'homme avec le monde, les objets et les «autres» qui l'environnent, semble guider la dynamique progressiste de notre développement technologique.

Adam Greenfield, dans son célèbre ouvrage de vulgarisation sur l'informatique ubiquitaire, Everyware (2007), pouvait ainsi affirmer: "Le corps humain est à la fois une riche source d'information et le véhicule au travers duquel nous percevons le monde et en cela il est probablement inévitable que tôt ou tard quelqu'un pense à la transformer en une forme de ressource mise en réseau.»

Le programme du Human Dynamics Laboratory du MIT, dirigé par Alex Pentland, l'un des ingénieurs infor- matiques les plus cités de la planète (Greenberg, 2010), offre une illustration frappante de cette tendance. À l'aide de capteurs "sociométriques» embarqués dans nos vêtements et capables d'analyser et d'interpréter en temps réel le comportement non verbal in situ de notre interlocuteur, nous serions en mesure de faire émerger ce que les éthologues appellent «l'honnêteté du signal», c'est-à-dire le sens effectif, actuel et performatif des messages. Jusqu'alors opacifiée par les distorsions de la médiation symbolique manifestant notre impuissance à saisir la complexité de cet énigmatique autre, la "vérité » des messages mise à nue par l'analyse algorithmique de ses comportements informationnels ouvrirait vers une communication pleine et transparente.

Bien évidemment, ces dispositifs sociométriques ne peuvent être pensés indépendamment de leur intégration dans un plus large appareillage technologique en réseau offrant la perspective d'une interprétation toujours plus fine des comportements. Les sociomètres feraient émerger, à travers ces opérations de data mining - auquel Alex Portland préfère le terme significatif de reality mining (Sanders, 2011) - des " patterns » interactionnels, sortes de structures inscrites au fondement de nos relations à l'autre. Ces dispositifs permettraient d'élaborer des «schémas prédictifs » qui guideraient nos décisions et qui éradiqueraient, à terme, l'inexorable tendance entropique qui régit nos rapports sociaux.

Pour Alex Pentland (2010), il «est clair que les instruments de mesure sociologique comme les sociomètres changeront nos conceptions traditionnelles de l'intelligence individuelle et de nos relations avec la société. Nous commençons à réaliser que nous ne partageons plus grand-chose avec l'être idéalisé et rationnel imaginé par la philosophie des Lumières. L'idée que notre conscience et notre pensée individuelle sont les facteurs clefs déterminant notre comportement en vient à être considérée comme la naïveté vaniteuse qui consistait à nous penser comme le centre de l'univers». 
Si cette fiction de l'autonomie de notre conscience individuelle était nécessaire dans un temps où régnait l'ignorance scientifique, il apparaît clair aux yeux de Portland que le dévoilement des mécanismes interactionnels par les sociomètres sonnerait le glas de notre humanisme narcissique. Nous serions enfin obligés de reconnaître l'ineptie de nos croyances en une quelconque intériorité mythique et, plus encore, en cette altérité radicale de l'Autre qu'Emmanuel Levinas (1993) situait au fondement de notre existence humaine. La transcendance immanente à nos rapports intersubjectifs, à cet Autre qui n'est pas moi et qui questionne à travers son visage ma propre existence, ne serait que pure illusion.

Le projet des Google Glass, dispositif de réalité augmentée implémenté dans des lunettes, pourrait bien constituer l'interface privilégiée de ce genre de "service» ou d'application ${ }^{5}$ destiné à "simplifier nos vies et nos relations». Ces lunettes, ou smart glasses, qui devraient arriver sur le marché dans le courant de l'année 2014, apparaissent comme la matérialisation archétypale de ce que pourrait être l'interface «invisible» du futur transformant progressivement nos corps en «terminaux bioélectroniques». Munis de ce dispositif: "Que se passera-t-il quand on nous offrira une véritable abondance de faits concernant cette personne - son niveau d'entendement, son réseau de connaissance, l'acidité de sa salive - et que ces données, provenant de sources jusqu'alors inaccessibles, apparaîtront de façon visuelle, projetées à la surface de nos lunettes? [...] Et que se passera-t-il quand l'image de nous-mêmes qui sera ainsi composée et proposée à autrui ne sera pas conforme à la manière dont nous aimerions être présentés?» (Greenfield, 2007) Travestissant ses espoirs en fatalité, c'est une inexorable transparence des rapports sociaux que Greenfield voit poindre.

Mais que dire d'un rapport intersubjectif médié par la production itérative de métadonnées extraites en temps réel du contexte à la fois comportemental, physiologique et historique de son interlocuteur ${ }^{6}$ ? Si nous déléguons, même inconsciemment, nos capacités d'entendement irréductiblement partielles et partiales, à la soi-disant objectivité des algorithmes d'analyse et d'interprétation, pouvonsnous penser notre rapport à l'autre autrement que par rapport à nous-même? Dans ce rapport à l'altérité s'effacerait l'ipséité, l'identité propre du sujet, au profit d'une «mêmeté» enfin dévoilée par les multiples "patterns» comportementaux qui régissent nos conditions d'"être au monde». Enfermé dans un comportementalisme informationnel étriqué, «chaque sujet est une multitude, mais il est multiple sans altérité, fragmentée en quantité de profils qui tous, se rapportent à "lui-même", à ses propensions, ses désirs présumés, ses opportunités et ses risques» (Rouvroy, 2013).

\section{Renaissance cybernétique}

Le projet ubiquitaire ne semble pas tant proposer une nouvelle téléologie technicienne qu'actualiser, dans des conditions sociotechniques radicalement différentes, les utopies de la communication qui innervaient l'entreprise technoscientifique de la première cybernétique. La symbiose homme-machine (Licklider, 1960); l'hypothèse d'une sous-traitance de notre "pensée algorithmique» aux technologies de traitement de l'information (Aurel, 1965); l'horizon d'une gouvernabilité cybernétique où les hommes délégueraient progressivement leur pouvoir de contrôle sur eux-mêmes et sur le monde aux «machines à communiquer», continue d'alimenter la dynamique $\mathrm{du}$ projet ubiquitaire. Plus que jamais, la vision d'un homme réduit à la somme de ses comportements informationnels semble guider souterrainement les trajectoires empruntées par l'industrie informatique. Des trajectoires, aussi diverses soient-elles, qu'il me semble possible de comprendre à partir des propositions théoriques et programmatiques de l'informatique ubiquitaire. Cette vision 
d'un écosystème numérique intégré "sans couture» au monde opérationnalisant nos relations aux autres et aux choses n'est pas non plus sans réveiller les craintes qui animaient déjà le père de la cybernétique à la fin des années 1950. À la fin de son célèbre ouvrage The Human Uses of Human Being, Norbert Wiener (1971) avait mis en garde ses lecteurs sur l'hypothétique émergence d'un Léviathan technologique, figure de l'hétéronomie technique incarnant progressivement une norme externalisée, une instance de régulation suprême aux commandes de nos destinées. Pour Wiener, le salut n'était pas tant dans cette «mega machine» que dans l'usage qu'en feraient les hommes. Plus important encore, écrivait-il, il faudra engager "quelques anthropologues sérieux» (Ibid.) dans sa conception qui pourraient garantir son adaptabilité au

\section{NOTES}

1. Les travaux très influents dans le champ des IHM de ses collègues Lucy Suchman et John Seely Brown sur l'action ou la cognition située vont fournir le cadre conceptuel, souvent implicite, de ce renversement.

2. Le protocole «Ethernet» inventé par Robert Metcalfe à la fin des années 1970 au PARC se référait déjà explicitement à l'éther pour qualifier l'omniprésence des flux informationnels dans les réseaux informatiques.

3. Une architecture supportée par la mise en œuvre du standard EPC (Electronic Product Code, consistant en une puce RFID - Radio Frequency Identification Tag) en passe de remplacer l'UPC (Universal Product Code: le traditionnel code barre) sous la direction scientifique de l'Auto ID-Lab du MIT qui a forgé le terme «Internet des objets». milieu humain. C'est bien, semble-t-il, cette inclinaison qui semble caractériser le design contemporain des interfaces homme-machine mobilisant aujourd'hui abondement les sciences humaines et sociales, alibis d'une téléologie technicienne toujours attachée à accroître notre désir de contrôle sur les termes de notre réalité humaine et matérielle. «Nous avons étudié le comportement humain - écrivait Alex Portland - et nous sommes maintenant entrain d'apprendre comment le façonner»(Greenberg, 2010). C'est sur ce consensus imaginaire d'une nature humaine enfin dévoilée par la technoscience et les sciences cognitives que se démultiplient aujourd'hui des programmes de $\mathrm{R} \& \mathrm{D}$ informatiques destinés à parachever, dans un écho à la fois fidèle et silencieux, l'entreprise démiurgique qui animait déjà la cybernétique au milieu du $\mathrm{xx}^{\mathrm{e}}$ siècle.

4. Paradigme dominant aujourd'hui dans le design des interfaces utilisateurs («UX design») qui doit beaucoup aux écrits de Donald Norman, Mark Weiser ou encore Lucy Suchman.

5. Une des nombreuses applications en développement pour ce dispositif est un système de reconnaissance faciale, sorte «d'assistant cognitif personnel» permettant aux individus atteints de prosopagnosie (agnosie visuelle les rendant incapable de mémoriser des visages) d'identifier leurs interlocuteurs. Mais cette application pourrait vite déborder le cadre purement thérapeutique...

6. C'est ce que met en scène un court-métrage israélien d'anticipation scientifique, intitulé Sight. 


\section{R ÉFÉRENCES B I B L I O G R A P H I Q U E S}

ArrayA, A., "Questioning Ubiquitous Computing», in ACM, CSC '95 Proceedings of the 1995 ACM 23rd Annual Conference on Computer Science, New York, ACM, 1995, p. 230-237.

Aurel, D., La Cybernétique et l'humain, Paris, Gallimard, 1965.

BAUdrillard, J., Le Système des objets, Paris, Gallimard, 1968.

COOPER, G. et Bowers, J., "Representing the User: Notes on the Disciplinary Rhetoric of Human-Computer Interaction", in Thomas, P. (dir.), The Social and Interactional Dimensions of Human-Computer Interfaces, Cambridge, Cambridge University Press, 1995, p. 48-66.

Denning, P. J., The Invisible Future. The Seamless Integration of Technology Into Everyday Life, New York, McGraw-Hill, 2002.

Dertouzos, M., The Unfinished Revolution: Human-Centered Computers and What They Can Do For Us, New York, HarperCollins, 2001.

Dourish, P. et Bell, G., Divining a Digital Future. Mess and Mythology in Ubiquitous Computing, Cambridge, MIT Press, 2011.

GAUCHet, M., Le Désenchantement du monde. Une histoire politique de la religion, Paris, Gallimard, 1985.

Gershenfeld, N., When Things Start to Think, Londres, Hodder and Stoughton, 1999.

Greenberg, A., "Mining Human Behaviour at MIT», Forbes, 12 août 2010 .

GREenfield, A., Everyware, la révolution de l'ubimédia, Limoges, FYP éditions, 2007.

Gross, N., "The Earth Will Don an Electronic Skin», Business Week, 30 août 1999

Levinas, E., Dieu, la mort et le temps, Paris, Grasset, 1993.

LiCklider, J. C. R., "Man-Computer Symbiosis», IRE Transactions on Human Factors in Electronics, vol. HFE-1, 1960, p. 4-11.

Norman, D. A., The Invisible Computer, Cambridge, MIT Press, 1998.

O’Reilly, T. et BAtTelle, J., Web Squared: Web 2.0 Five Years On, 2009, document numérique. Disponible en ligne sur: <assets. en.oreilly.com/1/event/28/web2009_websquared-whitepaper.pdf>, consulté le $07 / 01 / 2014$.
PUCHEU, D., «Le projet ubiquitaire: une nouvelle eschatologie informationnelle?", in BRATOSIN, S. et TUdor, M. A. (dir.), Actes du colloque international "Communication du symbolique et symbolique de la communication dans les sociétés modernes et postmodernes", ORC IARSIC - Essachess, 8-9 nov. 2012, Béziers, Iasi (Roumanie), Institutul European, coll. «Colloquia», 2013, p. 547-561.

PuCheu, D., Religiosité(s) technologiques américaine, Rennes, Apogée, à paraître [2014].

Pentland, S. A., Honest Signals. How They Shape Our World, Cambridge, MIT Press, 2010.

RHeingold, H., Smart Mobs. The Next Social Revolution. Transforming Cultures and Communities in the Age of Instant Access, New York, Perseus Book, 2002.

Rouvroy, A. et BERns, T., «Le nouveau pouvoir statistique », Multitudes, $\mathrm{n}^{\circ} 40,2010$, p. 88-103.

SANDERS, T., "Positive Computing», in BISWAS-DIEnER, R. (dir.), Positive Psychology as Social Change, New York, Springer, 2011, p. 309-327.

SAVARY, C., "L'au-delà de la technique et de son autre", in CANTIN, S. et MAger, R. (dir.). L'Autre de la technique. Perspectives multidisciplinaires, Québec-Paris, Presses de l'université Laval-L'Harmattan, 2000, p. 221-257.

Simondon, G., Du mode d'existence des objets techniques, Paris, Aubier, 1989.

WEISER, M., "The Computer for the 21st century ", Scientific American, vol. 265, n 3, 1991, p. 94-104.

WEISER, M., «The World Is Not a Desktop», Interactions, vol. 1, $\mathrm{n}^{\circ} 1,1994$, p. $7-8$.

Weiser, M. et Brown, J. S., «The Coming Age of Calm Technology", in Denning, P. J. et Metcalfe, R. M., Beyond Calculation. The Next 50 Years of Computing, New York, Copernicus - Springer, 1997, p. 75-85.

WEISER, M., GOLD, R. et Brown, J. S., «The Origins of Ubiquitous Computing Research at PARC in the Late 1980s ", IBM Systems Journal, vol. $38, \mathrm{n}^{\circ} 4,1999$, p. 693-696.

Wiener, N., Cybernétique et société, Paris, éditions des deux rives, 1971. 